\title{
25 Research Soure \\ The Role of USP6 Gene Rearrangement by FISH Analysis in Nodular Fasciitis and Histological Mimics
}

\section{Yanfen Feng}

Sun Yat-sen University Cancer Center

\section{Yu Zhang}

Sun Yat-sen University Cancer Center

Junpeng Lai

Sun Yat-sen University Cancer Center

\section{Zichen Zhang}

Sun Yat-sen University Cancer Center

\section{Min Li}

Sun Yat-sen University Cancer Center

\section{Huilan Rao}

Sun Yat-sen University Cancer Center

Yingchun Zhang ( $\nabla$ zhangyingch@sysucc.org.cn )

Sun Yat-sen University Cancer Center

\section{Research}

Keywords: USP6, Nodular fasciitis, fluorescence in situ hybridization

Posted Date: July 21st, 2021

DOI: https://doi.org/10.21203/rs.3.rs-728808/v1

License: (9) (1) This work is licensed under a Creative Commons Attribution 4.0 International License. Read Full License 


\section{Abstract}

Background: Nodular fasciitis (NF) is a common benign fibroblast and myofibroblast tumor. Although there have been constant concerns about its histological features and differential diagnosis, NF is still the most easily misdiagnosed mesenchymal lesions, especially over diagnosed as a sarcoma. USP6 gene rearrangement was reported recently to be a reproducible and specific gene change in NF, which strongly assists the diagnosis of NF. We aim to study USP6 Gene Rearrangement in NF and other spindle cell lesions mimicking NF, and to investigate the use of USP6 Gene Rearrangement in differentiating NF and its mimics.

Methods: USP6 translocation by fluorescence in situ hybridization (FISH) was studied on 40 cases of NF and 54 cases of other spindle cell lesions (including 10 cases of spindle cell sarcoma) that mimic NF.

Results: Thirty-four cases (85.5\%) of NF showed USP6 gene translocation, including 10 cases which previously diagnosed or considered as malignant diseases by the referring pathologists. All of other 54 cases of spindle cell lesions were negative for USP6 translocation.

Conclusions: USP6 gene rearrangement by FISH analysis is a reliable test for diagnosis of NF, and also a helpful adjunct to prevent over diagnosis of NF. Positive USP6 gene rearrangement by FISH could be a useful marker to exclude malignant lesions that mimics NF.

\section{Introduction}

NF is a commonly encountered, benign fibroblast and myofibroblast neoplasm, which usually presents as a small but rapidly growing mass ${ }^{1-3}$. It predominant occurs in young to middle-aged adults between 20 and 40 years, without sexual predisposition. Upper extremities are the most common affected site, followed by the trunk and head and neck. Cases occur in extremities and hands and feet are infrequent ${ }^{2-3}$. Most cases are located in the superficial subcutaneous fascia, but some can involve deep muscle tissues regardless of its location. Histologically, NF displays spindle cells with myofibroblasts features arranged into loose fascicles, showing storiform or S-like appearance. The spindle cells are usually bland looking, with variable myxoid vascularized to densely sclerotic stroma, and erythrocyte extravasations foci, with frequent foci of erythrocyte extravasations. These histological features of NF make it easily misdiagnosed as other spindle cell malignant tumors, such as lowgrade myofibroblastic sarcoma, myxofibrosarcoma, synovial sarcoma, and so on, which is harmful for the patients with risk of overtreatment. On the other hand, it should also be differentiated from benign or intermediate lesions such as desmoid fibromatosis, inflammatory myofibroblastic tumor, dermatofibroma, etc. Despite being recognized for over six decades and heightened awareness of this entity constantly, NF is still among the most common benign mesenchymal lesions misdiagnosed as a sarcoma. A recent report showed that the misdiagnosis rate of NF was more than $50 \%$ and the over diagnosis rate was more than $30 \%$. Making the diagnosis accurately and avoid over diagnosis of NF is still a great challenge for surgical pathologists, due to its confusing morphology and non-specific immunophenotype.

USP6 gene, also known as TRE17, encodes an ubiquitin-specific protease (ubiquitin-specific peptidase 6). USP6 protein is involved in regulation of ubiquitination and transport. It may also act as a driving oncogene due to gene rearrangement. A clear association between nodular fasciitis and USP6 gene rearrangement had been established. Molecular findings showed that MYH9-USP6 translocation is the most common rearrangement in NF. This finding resulted in its redefinition as a benign true neoplasm. In 2013, Amary et al conducted a series of studies identified 
USP6 rearrangements by FISH in 31 of 34 NF cases, with a sensitivity of about 91\%. Patel and colleagues reported similar findings, identified USP6 gene rearrangements in 20 of 26 NF cases. These results suggest that USP6 gene rearrangement test may serve as an important adjunct in the diagnosis and differential diagnosis of NF.

Up till now, only few literatures studied the USP6 gene rearrangement in NF and its mimics. Herein, we collected a cohort of NF and a group of its mimics, reviewed the clinical pathological features and morphology, and studied the USP6 rearrangement by FISH, aiming to analyze the use of USP6 rearrangement by FISH in diagnosis and differential diagnosis of NF.

\section{Materials And Methods}

\section{Cases selection}

Spindle lesions which diagnosed as NF, or have been mentioned as differentiated with NF, or have been tested USP6 gene translocation by FISH in the department of pathology, Sun Yat-sen University Cancer center from August 2017 to January 2020 were collected. To ensure the accuracy of the diagnosis, two senior pathologists specified in soft tissue (Yingchun Zhang and Huilan Rao) reviewed all the collected cases with initial routine hematoxylin and eosin (HE) and immunohistochemistry (IHC) slides. Some cases were also submitted to molecular test to confirm or exclude the corresponding lesions, for example, SS18-SSX fusion gene FISH analysis for synovial sarcoma, COL1A1-PDGFB fusion gene for protuberant dermatofibrosarcoma, etc. Following criteria of the World Health Organization (WHO, 2013), we divided those cases into three groups: NF, benign or intermediate lesions similar to NF, and malignant tumors similar to NF. Patients' age and sex, tumor location, related clinical information and follow-up data were collected. Patients diagnosed as nodular fasciitis and malignant tumors were followed up in detail. Sun Yat-sen University cancer center (SYSUCC) institutional ethics committee approved this study.

\section{FISH analysis}

FISH analysis for USP6 rearrangement was performed formalin-fixed, paraffin-embedded (FFPE) sections by using specific Dual Color Break Apart Probes following the manufacturer's instructions (LBP Medicine Science and Technology Co., Ltd, Guangzhou, China). The FISH signals were assessed under an Olympus BX 51 microscope (Olympus, Japan) equipped with a triple-pass filter (DAPI/Green/Orange, Vysis) Images were acquired using the Cell Sens Standard Imaging System (version 1.7, Olympus Microsystems Inc., Japan). USP6 signals were independently scored by two experienced pathologists (and). Cells with an orange and green signal were scored as normal. The distance of break-apart signals between one of the signals, and one orange and green signal were more than twice, indicating a rearrangement for the USP6 gene. A total of 100 tumor cells were counted and the percentage of the cells with split signal was recorded. Positive cases with a rearrangement for the USP6 gene were defined as those with $>10 \%$ of cells showing the break-apart signal.

\section{Results}

\section{General clinical and demographic features of the total cohort}

After eliminating the cases of duplication and failure in detection, 94 cases of spindle cell lesions were retrieved, including 40 cases of NF, 44 cases of benign or intermediate lesions and 10 cases of malignant tumors 
histologically similar to NF. The cohort consisted of 52 males (55.3\%) and 42 females (44.7\%), ranging in age from 1 to 70 years (median, 30 years). The patient's clinico-pathological information is shown in Table 1 and Table 2. 
Table 1

Clinico-pathological features of nodular fasciitis cases

\begin{tabular}{|c|c|c|c|c|c|c|c|}
\hline $\begin{array}{l}\text { Case } \\
\text { no. }\end{array}$ & Age & Sex & Site & $\begin{array}{l}\text { Duration } \\
\text { time } \\
\text { before } \\
\text { diagnosis }\end{array}$ & Size(cm) & previous diagnosis & $\begin{array}{l}\text { USP6 gene } \\
\text { rearrangement } \\
\text { by FISH }\end{array}$ \\
\hline 1 & 33 & $M$ & Right neck & 10 days & 2.5 & In-house case & Positive \\
\hline 2 & 42 & M & $\begin{array}{l}\text { Subcutis of } \\
\text { right forearm }\end{array}$ & 1 week & 2 & In-house case & Positive \\
\hline 3 & 42 & $\mathrm{~F}$ & Right thigh & 1 month & 2.3 & In-house case & Positive \\
\hline 4 & 26 & $\mathrm{~F}$ & $\begin{array}{l}\text { Right lower } \\
\text { abdominal wall }\end{array}$ & NA & 1.3 & In-house case & Positive \\
\hline 5 & 44 & $\mathrm{~F}$ & Left hip & NA & 1.7 & In-house case & Positive \\
\hline 6 & 57 & M & $\begin{array}{l}\text { Right } \\
\text { sternoclavicular } \\
\text { joint }\end{array}$ & NA & 4.0 & In-house case & Positive \\
\hline 7 & 27 & $\mathrm{~F}$ & Right thigh & NA & 2.2 & In-house case & Positive \\
\hline 8 & 46 & $\mathrm{~F}$ & $\begin{array}{l}\text { Lower left } \\
\text { clavicle }\end{array}$ & 6 months & 3.2 & In-house case & Positive \\
\hline 9 & 13 & $\mathrm{~F}$ & $\begin{array}{l}\text { Right thigh root } \\
\text { intramuscular }\end{array}$ & 1 year & 6.2 & In-house case & Positive \\
\hline 10 & 60 & $M$ & Left chest wall & $\begin{array}{l}\text { half a } \\
\text { month }\end{array}$ & 2.0 & Spindle cell tumor & Positive \\
\hline 11 & 26 & M & Left upper arm & NA & NA & Dermatofibroma & Positive \\
\hline 12 & 4 & M & Waist back & 1 day & 4.8 & $\begin{array}{l}\text { Desmoid-type } \\
\text { fibromatosis }\end{array}$ & Positive \\
\hline 13 & 50 & M & Right cheek & 4 months & NA & $\begin{array}{l}\text { Inflammatory } \\
\text { myofibroblastic tumor }\end{array}$ & Positive \\
\hline 14 & 30 & $M$ & Left cheek & 2 weeks & NA & Nodular fasciitis & Positive \\
\hline 15 & 5 & $M$ & Eyelid & NA & 1.0 & $\begin{array}{l}\text { Spindle cell tumor, } \\
\text { borderline tumor is } \\
\text { considered }\end{array}$ & Positive \\
\hline 16 & 7 & $M$ & Left waist & $\begin{array}{l}\text { half a } \\
\text { year }\end{array}$ & 1 & Nodular fasciitis & Positive \\
\hline 17 & 45 & $\mathrm{~F}$ & Right thumb & NA & 1.5 & Soft tissue tumor & Positive \\
\hline 18 & 34 & M & Nose Root & $\begin{array}{l}\text { half a } \\
\text { month }\end{array}$ & NA & $\begin{array}{l}\text { Desmoid-type } \\
\text { fibromatosis }\end{array}$ & Positive \\
\hline 19 & 57 & M & Right eye orbit & 1 month & 1.0 & Haemangioendothelioma & Positive \\
\hline 20 & 32 & $\mathrm{~F}$ & Left cheek & 2 months & NA & NA & Positive \\
\hline
\end{tabular}




\begin{tabular}{|c|c|c|c|c|c|c|c|}
\hline $\begin{array}{l}\text { Case } \\
\text { no. }\end{array}$ & Age & Sex & Site & $\begin{array}{l}\text { Duration } \\
\text { time } \\
\text { before } \\
\text { diagnosis }\end{array}$ & Size $(\mathrm{cm})$ & previous diagnosis & $\begin{array}{l}\text { USP6 gene } \\
\text { rearrangement } \\
\text { by FISH }\end{array}$ \\
\hline 21 & 3 & M & Left forehead & $\begin{array}{l}\text { half a } \\
\text { year }\end{array}$ & 1.2 & Soft tissue tumor & Positive \\
\hline 22 & 29 & $M$ & $\begin{array}{l}\text { Side of Cervical } \\
\text { spine }\end{array}$ & 4 months & 3.0 & Myofibroblastic tumor & Positive \\
\hline 23 & 30 & $\mathrm{~F}$ & Left back & 1 week & 2.5 & Nodular fasciitis & Positive \\
\hline 24 & 7 & M & Right temporal & 5 months & 1 & $\begin{array}{l}\text { Inflammatory } \\
\text { myofibroblastic tumor }\end{array}$ & Positive \\
\hline 25 & 53 & $\mathrm{~F}$ & Left biceps & 1 month & 2.4 & leiomyosarcoma & Positive \\
\hline 26 & 54 & M & Left hand & 1 year & NA & Synovial sarcoma & Positive \\
\hline 27 & 4 & M & $\begin{array}{l}\text { Right parotid } \\
\text { region }\end{array}$ & 1 month & 1.7 & Infantile fibrosarcoma & Positive \\
\hline 28 & 48 & M & Waist & NA & NA & $\begin{array}{l}\text { Dermatofibrosarcoma } \\
\text { protuberans }\end{array}$ & Positive \\
\hline 29 & 11 & M & Posterior neck & 3 months & 3.0 & $\begin{array}{l}\text { Dermatofibrosarcoma } \\
\text { protuberans }\end{array}$ & Positive \\
\hline 30 & 36 & $\mathrm{~F}$ & Left forearm & 3 weeks & 3.5 & $\begin{array}{l}\text { Low-grade fibromyxoid } \\
\text { sarcoma }\end{array}$ & Positive \\
\hline 31 & 15 & M & Right neck & 3 months & 3.0 & $\begin{array}{l}\text { Low-grade fibromyxoid } \\
\text { sarcoma }\end{array}$ & Positive \\
\hline 32 & 22 & M & Buccal & NA & 1.0 & $\begin{array}{l}\text { Low-grade fibromyxoid } \\
\text { sarcoma }\end{array}$ & Positive \\
\hline 33 & 44 & $\mathrm{~F}$ & Right hip & 3 months & 2.8 & $\begin{array}{l}\text { Low-grade fibromyxoid } \\
\text { sarcoma }\end{array}$ & Positive \\
\hline 34 & 34 & M & Right thigh & 10 days & 4.0 & $\begin{array}{l}\text { Myxofibrosarcoma / } \\
\text { Low-grade fibromyxoid } \\
\text { sarcoma }\end{array}$ & Positive \\
\hline 35 & 35 & M & $\begin{array}{l}\text { Extrathyroid } \\
\text { soft tissue } \\
\text { nodule }\end{array}$ & NA & NA & $\begin{array}{l}\text { Postoperative thyroid } \\
\text { cancer }\end{array}$ & Negative \\
\hline 36 & 36 & $\mathrm{~F}$ & Right thigh & $>2$ years & 1.4 & NA & Negative \\
\hline 37 & 31 & $\mathrm{~F}$ & $\begin{array}{l}\text { Subcutis of } \\
\text { right upper arm }\end{array}$ & NA & 1.2 & $\begin{array}{l}\text { In the course of breast } \\
\text { cancer treatment }\end{array}$ & Negative \\
\hline 38 & 25 & $M$ & Right chest wall & NA & NA & NA & Negative \\
\hline 39 & 48 & M & Right thigh & 1 week & 2.0 & Nodular fasciitis & Negative \\
\hline 40 & 5 & $\mathrm{~F}$ & $\begin{array}{l}\text { Subcutaneous } \\
\text { back }\end{array}$ & $\begin{array}{l}>5 \\
\text { months }\end{array}$ & NA & NA & Negative \\
\hline
\end{tabular}


Page $7 / 17$ 
Table 2

clinico-pathological features of lesions mimicking NF

\begin{tabular}{|c|c|c|c|c|c|}
\hline $\begin{array}{l}\text { Case } \\
\text { no. }\end{array}$ & Tumor & Age & Sex & Site & $\begin{array}{l}\text { USP6 gene } \\
\text { rearrangement by FISH }\end{array}$ \\
\hline 1 & Desmoid-type fibromatosis & 31 & M & Cervical vertebra & Negative \\
\hline 2 & Desmoid-type fibromatosis & 34 & $\mathrm{~F}$ & $\begin{array}{l}\text { the left lower } \\
\text { abdominal wall }\end{array}$ & Negative \\
\hline 3 & Desmoid-type fibromatosis & 23 & $\mathrm{~F}$ & Left neck & Negative \\
\hline 4 & Desmoid-type fibromatosis & 65 & $M$ & $\begin{array}{l}\text { Right abdominal } \\
\text { paraaortic }\end{array}$ & Negative \\
\hline 5 & Desmoid-type fibromatosis & 48 & M & Left neck & Negative \\
\hline 6 & Desmoid-type fibromatosis & 42 & $\mathrm{~F}$ & Deep fasciatus & Negative \\
\hline 7 & Desmoid-type fibromatosis & 61 & $M$ & Right shoulder & Negative \\
\hline 8 & Desmoid-type fibromatosis & 30 & $\mathrm{~F}$ & $\begin{array}{l}\text { Left rectus } \\
\text { abdominis }\end{array}$ & Negative \\
\hline 9 & Desmoid-type fibromatosis & 22 & $\mathrm{~F}$ & Left breast & Negative \\
\hline 10 & Desmoid-type fibromatosis & 20 & $M$ & Right upper arm & Negative \\
\hline 11 & Desmoid-type fibromatosis & 15 & $\mathrm{~F}$ & Left abdominal wall & Negative \\
\hline 12 & Desmoid-type fibromatosis & 29 & $\mathrm{~F}$ & Groin & Negative \\
\hline 13 & Desmoid-type fibromatosis & 18 & $\mathrm{~F}$ & Left upper arm & Negative \\
\hline 14 & Desmoid-type fibromatosis & 33 & $\mathrm{~F}$ & Right upper jaw & Negative \\
\hline 15 & Desmoid-type fibromatosis & 53 & $\mathrm{~F}$ & Left abdominal wall & Negative \\
\hline 16 & Desmoid-type fibromatosis & 67 & $\mathrm{~F}$ & Right chest wall & Negative \\
\hline 17 & Desmoid-type fibromatosis & 28 & $\mathrm{~F}$ & abdominal cavity & Negative \\
\hline 18 & Desmoid-type fibromatosis & 25 & $\mathrm{~F}$ & NA & Negative \\
\hline 19 & $\begin{array}{l}\text { Reactive/myofibroblast } \\
\text { proliferative lesions }\end{array}$ & 24 & M & Neck shoulder & Negative \\
\hline 20 & $\begin{array}{l}\text { Reactive/myofibroblast } \\
\text { proliferative lesions }\end{array}$ & 46 & M & Right thigh & Negative \\
\hline 21 & $\begin{array}{l}\text { Reactive/myofibroblast } \\
\text { proliferative lesions }\end{array}$ & 16 & $M$ & Right tibia & Negative \\
\hline 22 & $\begin{array}{l}\text { Reactive/myofibroblast } \\
\text { proliferative lesions }\end{array}$ & 47 & M & $\begin{array}{l}\text { Left upper arm } \\
\text { intermuscular }\end{array}$ & Negative \\
\hline 23 & $\begin{array}{l}\text { Reactive/myofibroblast } \\
\text { proliferative lesions }\end{array}$ & 28 & M & Face & Negative \\
\hline 24 & $\begin{array}{l}\text { Reactive/myofibroblast } \\
\text { proliferative lesions }\end{array}$ & 54 & $\mathrm{~F}$ & Thyroid & Negative \\
\hline
\end{tabular}




\begin{tabular}{|c|c|c|c|c|c|}
\hline $\begin{array}{l}\text { Case } \\
\text { no. }\end{array}$ & Tumor & Age & Sex & Site & $\begin{array}{l}\text { USP6 gene } \\
\text { rearrangement by FISH }\end{array}$ \\
\hline 25 & $\begin{array}{l}\text { Reactive/myofibroblast } \\
\text { proliferative lesions }\end{array}$ & 29 & $\mathrm{~F}$ & Right neck & Negative \\
\hline 26 & Cellular fibrous histiocytoma & 14 & M & Left arm & Negative \\
\hline 27 & Cellular fibrous histiocytoma & 15 & M & Left abdominal wall & Negative \\
\hline 28 & Solitary fibrous tumor & 32 & $\mathrm{~F}$ & Abdominal wall & Negative \\
\hline 29 & Solitary fibrous tumor & 46 & M & Right nasal cavity & Negative \\
\hline 30 & Myositis ossificans & 70 & $\mathrm{~F}$ & Right calf & Negative \\
\hline 31 & Myositis ossificans & 20 & M & Left axillary fossa & Negative \\
\hline 32 & $\begin{array}{l}\text { Inflammatory myofibroblast } \\
\text { tumor }\end{array}$ & 55 & $\mathrm{~F}$ & $\begin{array}{l}\text { Left mandibular } \\
\text { gingiva }\end{array}$ & Negative \\
\hline 33 & Deep benign fibrous histiocytoma & 50 & M & NA & Negative \\
\hline 34 & Fibrohistiocytoma & 19 & $\mathrm{~F}$ & Right upper arm & Negative \\
\hline 35 & Proliferative myositis & 50 & $\mathrm{~F}$ & Right thigh & Negative \\
\hline 36 & Schwannoma & 37 & M & Left thigh & Negative \\
\hline 37 & perineurioma & 42 & M & Skin of left ankle & Negative \\
\hline 38 & Dermatofibroma & 41 & M & left waistwaist & Negative \\
\hline 39 & Benign myofibroblastic tumor & 26 & $\mathrm{~F}$ & Left forearm & Negative \\
\hline 40 & Benign myofibroblastic tumor & 20 & $\mathrm{~F}$ & $\begin{array}{l}\text { Subcutaneous of } \\
\text { right waist }\end{array}$ & Negative \\
\hline 41 & Benign or intermediate tumor & 1 & M & Left hip & Negative \\
\hline 42 & Fibrous dysplasia of bone & 25 & M & Left femoral neck & Negative \\
\hline 43 & Chondroblastoma & 20 & M & Upper right femur & Negative \\
\hline 44 & Chondroblastoma & 11 & $\mathrm{~F}$ & Upper left tibia & Negative \\
\hline 45 & $\begin{array}{l}\text { Low-grade myofibroblast } \\
\text { sarcoma }\end{array}$ & 28 & M & Right neck & negative \\
\hline 46 & $\begin{array}{l}\text { Low-grade myofibroblast } \\
\text { sarcoma }\end{array}$ & 16 & M & Left thigh & negative \\
\hline 47 & $\begin{array}{l}\text { Low-grade myofibroblast } \\
\text { sarcoma }\end{array}$ & 26 & M & Right neck & negative \\
\hline 48 & Low grade myxoid fibrosarcoma & 57 & $\mathrm{~F}$ & Left knee & negative \\
\hline 49 & $\begin{array}{l}\text { Pleomorphic undifferentiated } \\
\text { sarcoma }\end{array}$ & 43 & M & Right supraclavicle & negative \\
\hline 50 & Synovial sarcoma & 24 & $\mathrm{~F}$ & Right sole & negative \\
\hline 51 & osteosarcoma & 24 & $\mathrm{~F}$ & Right upper jaw & negative \\
\hline
\end{tabular}




\begin{tabular}{|llclll|}
\hline $\begin{array}{l}\text { Case } \\
\text { no. }\end{array}$ & Tumor & Age & Sex & Site & $\begin{array}{l}\text { USP6 gene } \\
\text { rearrangement by FISH }\end{array}$ \\
\hline 52 & osteosarcoma & 36 & M & Left distal femur & negative \\
\hline 53 & $\begin{array}{l}\text { Low-grade malignant } \\
\text { mesenchymal tumors }\end{array}$ & 29 & M & Subcutaneous back & negative \\
\hline 54 & Spindle cell rhabdomyosarcoma & 21 & M & $\begin{array}{l}\text { Anterior maxillary } \\
\text { region }\end{array}$ & negative \\
\hline
\end{tabular}

Of the NF group, 9 were in-house cases and 31 were consult cases, with the latter being the majority. The most common site affected is head and neck $(n=15,37.5 \%)$, followed by the trunk $(n=10,25.0 \%)$, upper extremities $(n=$ $7,17.5 \%$ ), and lower extremities ( $n=8,20.0 \%$ ). Tumor size ranged from 1.0 to $6.2 \mathrm{~cm}$ (median, $2.0 \mathrm{~cm}$ ). Tumor size of 30 cases was recorded, with 21 (73.3\%) cases smaller than $3 \mathrm{~cm}$. Median age was 32.5 years (ranging, 1-60 years). Twenty-five $(25 / 40,62.5 \%)$ patients were in their second to fifth decades, and 10 (10/40, 25.0\%) patients were children and adolescents (age $\leq 15$ years). The preoperative duration of 28 cases of patients were obtained, ranged from 1 day to > 2 years, $53.6 \%$ (15/28) of which were less than 3 months, while $28.6 \%(22 / 28)$ were less than 6 months. Thirty-one cases of NF were consult cases, and the initial submitted diagnoses and the reason for external consultation for 27 cases were recorded. Some of them were given definite diagnosis by the referring pathologists, while others with indefinite diagnosis were given assessment of potential biological behavior (e.g., favoring a sarcoma or sarcomatoid neoplasm). Of these 27 cases with diagnostic opinions, only 4 cases were correctly diagnosed as $\mathrm{NF}$, while $9(9 / 27,33.3 \%)$ cases were initially diagnosed as malignant tumors, including lowgrade malignant fibromyxoid sarcoma, myxoid fibrosarcoma, myxoid liposarcoma, synovial sarcoma, etc. Others were named as desmoid fibromatosis, inflammatory myofibroblast tumor and other benign or intermediate lesions.

Microscopically, our cases had a wide variety of morphologic features: many cases had abundant myxoid matrix, and a few cases had a higher cellularity, while some lesions was characterized by increased collagen production, sometimes with deposition of keloid-like collagen fibers. All the cases were composed of plump, immatureappearing fibroblasts and myofibroblasts that arranged in short intersecting fascicles. In the area with obvious mucoid degeneration, those cells were arranged in a haphazard fashion, often called "tissue culture appearance" or "feathery pattern", usually accompanied extravasated erythrocytes and scattered lymphocytes. Mitotic figures are fairly common, but cytological pleomorphism and nuclear atypia were absent. Most lesions were well circumscribed but unencapsulated, whereas a minority of cases had poorly defined borders with infiltration into the surrounding skeletal muscle, adipose tissue, and entrapped nerve fascicles. The stroma was at least focally myxoid in all cases and there were frequent cystic spaces in these myxoid areas. In some cases, the cells were abundant and dense, with more mitotic images and similar morphology to malignant tumors (Fig. 1). Mitotic figures are fairly common, but atypical mitoses are virtually never seen. Possibly related to the duration of the lesion, there are three major subtypes of NF: mucinous, cellular and fibrous.

Due to its variable morphologic features, nodular fasciitis were easily misdiagnosed as other benign of even malignant lesions with mucinous appearance, of with proliferation of fibroblasts and myofibroblasts, such as lowgrade myofibroblast sarcoma, desmoid-type fibromatosis, cellular fibrous histiocytoma, solitary fibrous tumor, myositis ossificans, inflammatory myofibroblast tumor, et al. 
Clinic-pathological features of 54 cases of spindle cell lesions mimicking NF were summarized in Table 2. Some of the cases displayed proliferation of fibroblast or myofibroblast features, some showed mucinous stroma, some showed proliferation of collagen fiber, and some showed dense spindle cellularity. All these features were mimicking different periods of NF, which have been described as mucinous, cellular and fibrous subtypes (Fig. 2).

\section{Frequency of USP6 translocation in NF and in NF-like lesions}

The results of FISH analysis of USP6 translocation was shown in Table 3. Of these 98 cases, 35 (35/98, 37.6\%) were positive. Within the 40 cases of NF, 35 (35/40, 85.0\%) of them were positive. All of other 58 cases that mimic NF were negativet. It is worth noting that all 9 cases that initially diagnosed as malignant tumors by the submitting pathologist were all positive for USP6 gene rearrangement. The microscopic images and FISH results of representative cases showed in Fig. 3.

Table 3

The frequency of USP6 Gene Rearrangement by FISH Analysis in nodular fasciitis and histological mimics

\begin{tabular}{|lll|}
\hline Lesions & cases (n) & USP6 gene rearrangement (n, \%) \\
\hline Nodular fasciitis & 40 & $34(85.0)$ \\
\hline Begnin or intermediate tumor & 45 & $0 / 45(0)$ \\
\hline Fibromatosis & 17 & $0 / 17$ \\
\hline Reactive/reparative myofibroblastic lesion & 7 & $0 / 7$ \\
\hline Myositis ossificans & 2 & $0 / 2$ \\
\hline Chondroblastoma & 2 & $0 / 2$ \\
\hline Others & 17 & $0 / 17$ \\
\hline Malignant tumor & 10 & $0 / 10$ \\
\hline Total & 95 & $34 / 95(35.8)$ \\
\hline
\end{tabular}

\section{Discussion}

In this study, we reviewed a cohort of NF and its histological mimics, collected their clinico-pathological characteristics, and studied their USP6 gene rearrangement status by FISH. Clinico-pathological analysis showed that NF occurred over a wide age range, from 1 to 60 years old, and predominant in young to middle-aged adults between 20 and 50 years old. Most of the lesions grow rapidly and the lesion duration was short, with most cases less than 3 months. The greatest dimensions of NF were smaller than $3 \mathrm{~cm}$ in $73.3 \%$ of cases. The follow-up data showed that most NF patients only underwent complete resection, and no recurrence or metastasis happened. These results demonstrated important clinical features of NF: rapid growth, small nodule, and benign course.

Similar to previously reported series, the pathologic spectrum of NF is wide and the morphology can vary significantly between and within cases. However, in the case of complete resection, more or less mucoid degeneration, or microcapsule structure with lymphocyte infiltration can be seen in the background of spindle tumor cells in most cases, suggesting that this is a very important diagnostic clue. Mitotic figures are fairly 
common, but atypical mitoses are virtually never seen. The absence of atypical mitosis is one of the most important morphological features to distinguish nodular fasciitis from malignant tumors.

It is worth noting that NF is very easy to be misdiagnosed, especially over diagnosed as a sarcoma. In 1991, Montgomery EA and Meis JM reviewed 53 cases of NF and found that the misdiagnosis rate was $57 \%$, of which $21 \%$ were over diagnosed as sarcomas. In 2018, Erber R and Agaimy A reported that of the 36 consult cases with diagnostic suggestions, only one third of NFs were correctly diagnosed by submitting pathologists, while one third was initially judged as malignant. Our data showed that only $9.6 \%$ of NF was correctly diagnosed by submitting pathologists. Over diagnosis as malignancy (ranging from low-grade to high-grade sarcoma) was rendered or favored in $35.4 \%$ of cases, which would cause catastrophic clinical consequences. The main reasons for over diagnosis attributed to not considering NF as a differential diagnosis, or mislead by features such as increased mitotic activity, high cellularity, high Ki-67 index, deep infiltrative location, prominent myxoid change, and good circumscription being not compatible with a diagnosis of NF. Since the histological features of NF is confusing, while immunohistochemistry staining is not helpful, sometimes even misleading, more objective and specific auxiliary methods are urgently needed.

In 2011, Erickson Johnson et al. found that USP6 gene rearrangement occurred in approximately 92\% (44/48) of NF cases, but not in normal control tissues or other spindle cell lesions that required to be differentiated from NF, suggesting that USP6 gene rearrangement was a reproducible and specific genetic change in NF, and has potential application value in clinical diagnosis. In recent studies conducted by Amary et al., USP6 rearrangement was detected by FISH in 31 of 34 NF cases with a sensitivity of approximately $91 \%$. Patel and colleagues reported similar findings, which identified USP6 gene rearrangements in 20 of 26 NF cases.

In our study, 34 of 40 NF cases are positive for USP6 FISH test, with a sensitivity of $85 \%$, consistent with the results reported in the literature. For those NF cases which were initially diagnosed as malignant tumors by the submitting pathologist, the USP6 gene rearrangement by FISH was 100\% positive (9/9), while for those malignant cases that needed to be differentiated from NF, including low-grade myxofibrosarcoma, synovial sarcoma, osteosarcoma and other cases, USP6 gene rearrangement by FISH was 100\% negative (10/10). Positive USP6 gene rearrangement by FISH could prompt the possibility of NF.

As showed in table 4, including our series, a total of 53 cases of different malignancies were tested by USP6 FISH and none was positive. Therefore, a positive of USP6 gene rearrangement test could almost rule out malignant tumors, which is of great value in preventing NF from being over diagnosed as sarcoma.

In summary, USP6 gene rearrangement by FISH analysis is a reliable test for the diagnosis of NF, and also a helpful adjunct to prevent over diagnosis of other malignancies. Negative USP6 gene rearrangement by FISH could not exclude NF, but USP6 gene rearrangement by FISH indicates that NF is the most likely, and the possibility of other malignant tumors is basically excluded. As the technology of USP6 gene rearrangement tested by FISH is mature and widely available, we recommend that USP6 FISH analysis should be performed to exclude NF and other USP6induced neoplasms before a diagnosis of malignant rendered when a case with rapidly growing, small nodule without definite atypical mitoses is encountered.

\section{Abbreviations}


NF: Nodular fasciitis; FISH: fluorescence in situ hybridization; HE: hematoxylin and eosin; IHC:

immunohistochemistry

\section{Declarations}

\section{Ethics approval and consent to participate}

This study was ethically approved by Sun Yat-sen University Cancer Center IRB, and has been granted exemption from requiring informed consent to participate from participants.

\section{Consent for publication}

Not applicable.

\section{Availability of data and materials}

The datasets generated during and/or analyzed for this study are available from the corresponding author upon reasonable request.

\section{Competing interests}

The authors declare that they have no competing interests.

\section{Funding}

Not applicable.

\section{Authors' contributions}

ZYC designed the study. FYF and ZYC drafted the manuscript and contributed to data analysis. ZYC and RHL contributed to the diagnoses. ZY contributed to clinico-pathological data collection. LJP assisted in patient selection and figure preparation. ZZC contributed to FISH analysis. LM contributed to the patients' follow-up. All authors read and approved the final manuscript.

\section{Acknowledgements}

Not applicable.

\section{References}

1. Goldblum JR FA, Weiss SW eds. Enzinger and Weiss's soft tissue tumors, 7th edn. Philadelphia, PA: Elsevier Saunders, 2020; 203-214.

2. Lazar A EH, Oliveira AM. Nodular fasciitis. In Fletcher CDM, Bridge JA, Hogendoorn PCW, Mertens F eds. World Health Organization classification of tumours of soft tissue and bone. Lyon: IARC Press, 2013; 46-47.

3. Prince EB, Silliphant WM, Shuman R. Nodular fasciitis: a clinicopathologic analysis of 65 cases. Am J Clin Pathol 1961;35:122-36.

4. Allen PW. Nodular fasciitis. Pathology 1972;4:9-26. 
5. Meister P, Bückmann FW, Konrad E. Nodular fasciitis (analysis of 100 cases and review of the literature). Pathol Res Pract 1978;162:133-65.

6. Lu, L, Lao, IW, Liu, X, Yu L, Wang, J.Nodular fasciitis: a retrospective study of 272 cases from China with clinicopathologic and radiologic correlation. Ann Diagn Pathol.2015 Jun ;19(3):180-5.

7. Lindberg MR. Diagnostic Pathology: Soft Tissue Tumors reP, PA: Elsevier Saunders, 2019; 114-119.

8. Erber R, Agaimy A. Misses and near misses in diagnosing nodular fasciitis and morphologically related reactive myofibroblastic proliferations: experience of a referral center with emphasis on frequency of USP6 gene rearrangements. Virchows Arch. 2018 Sep;473(3):351-360.

9. Oliveira AM, Chou MM. The TRE17/USP6 oncogene: a riddle wrapped in a mystery inside an enigma. Front Biosci (Schol Ed). 2012 Jan 1;4:321-34.

10. Oliveira AM, Chou MM. USP6-induced neoplasms: the biologic spectrum of aneurysmal bone cyst and nodular fasciitis. Hum Pathol. 2014 Jan;45(1):1-11.

11. Erickson-Johnson MR CM, Evers BR, Roth CW, Seys AR, Jin L, Ye Y, Lau AW, Wang X, Oliveira AM. Nodular fasciitis: a novel model of transient neoplasia induced by MYH9-USP6 gene fusion. Lab Invest. 2011 Oct;91(10):1427-33.

12. Amary MF YH, Berisha F, Tirabosco R, Presneau N, Flanagan AM. . Detection of USP6 gene rearrangement in nodular fasciitis: an important diagnostic tool. Virchows Arch. 2013 463: 97-8.

13. Rosenberg AE. Pseudosarcomas of soft tissue. Arch Pathol Lab Med. 2008 Apr;132(4):579-86.

14. Pichler Sekulic S, Sekulic M. Nodular fasciitis of the vulva: a challenging histopathologic diagnosis supported by the detection of USP6 gene rearrangement. APMIS. 2016 Jun;124(6):534-537.

15. Shimizu S HH, Enjoji M. Nodular fasciitis: an analysis of 250 patients. Pathology. 1984 Apr;16(2):161-6.

16. Patel NR, Chrisinger JSA, Demicco EG, Sarabia SF, Reuther J, Kumar E, Oliveira AM, Billings SD, Bovée JVMG, Roy A, Lazar AJ, Lopez-Terrada DH, Wang WL. USP6 activation in nodular fasciitis by promoter-swapping gene fusions. Mod Pathol. 2017 Nov;30(11):157

\section{Table}

Table 4 is not available with this version.

\section{Figures}




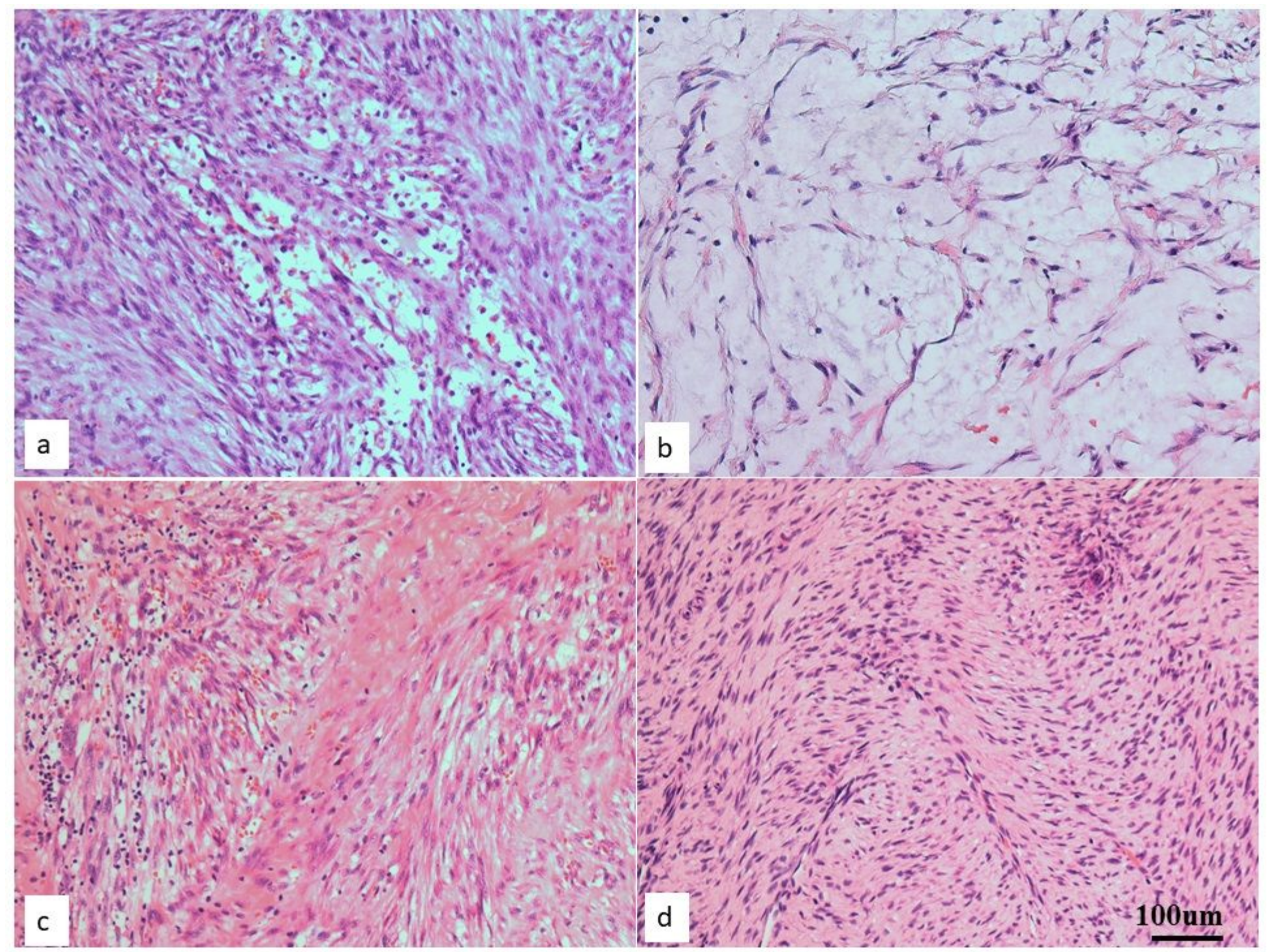

\section{Figure 1}

Different histological features of NF (H\&E, 400x). a. Plump, immature-appearing fibroblasts and myofibroblasts arranged in short intersecting fascicles. b. Area with obvious mucoid degeneration, and cells were arranged in a haphazard fashion (called "tissue culture appearance" or "feathery pattern). c. Extravasated erythrocytes and scattered lymphocytes infiltrate. d. Collagen production of stroma. 


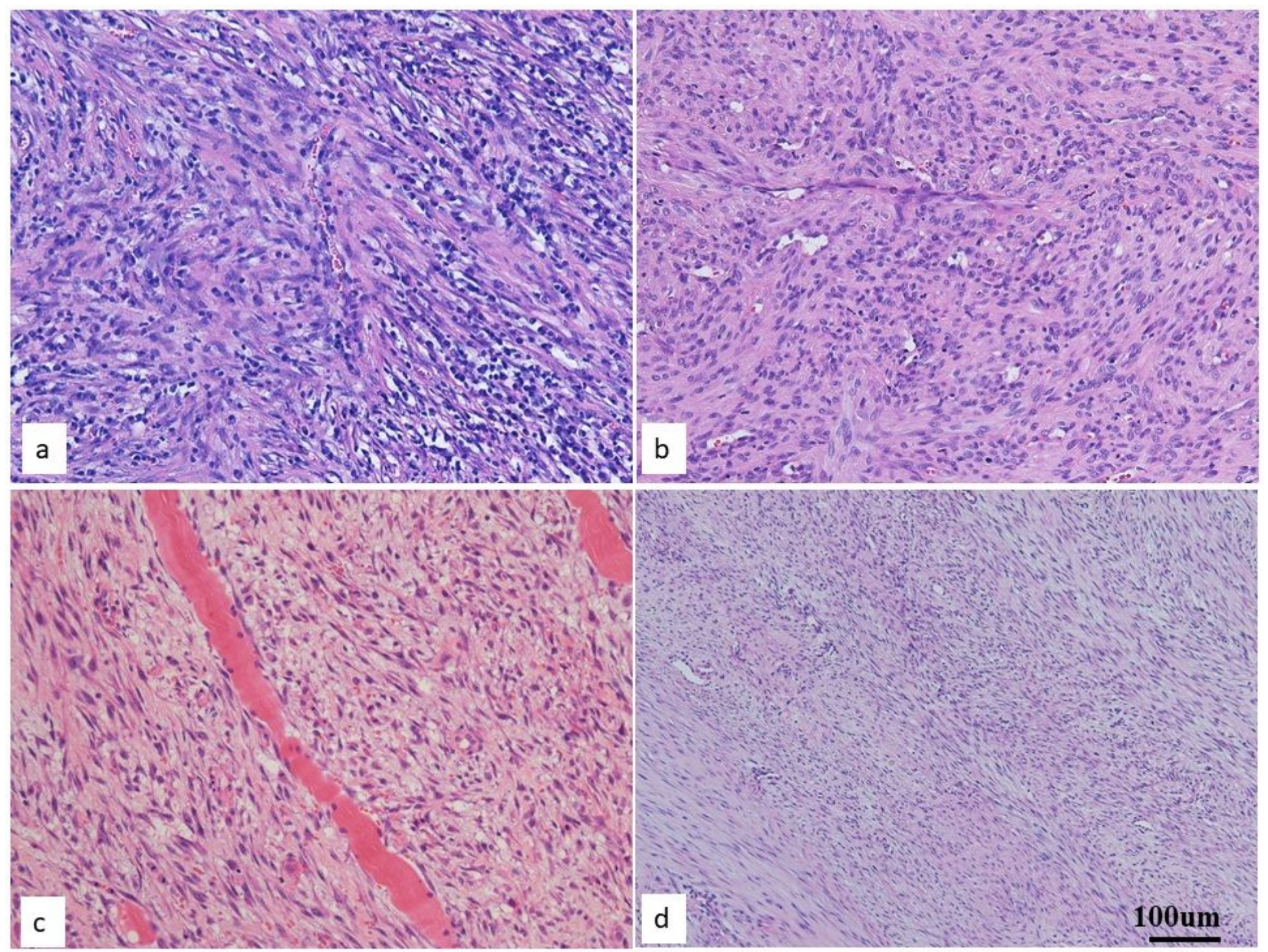

\section{Figure 2}

Different histological features of spindle cell lesions mimicking NF (H\&E, 400x). a. Inflammatory myofibroblast tumor showed myofibroblasts arranged in Fascicles, with numerous inflammatory cells. b. low-grade myofibroblast sarcoma showed fat spindle cells with abundant plasma arranged in fascicles. c. myositis ossificans showed long spindle cell range in fascicles, with abundant eosinophilic plasma, and bony tissues with osteoblast around were seen. d. desmoid-type fibromatosis showed fibroblasts and myofibroblasts arranged in fascicles, with collagen rich stroma. 


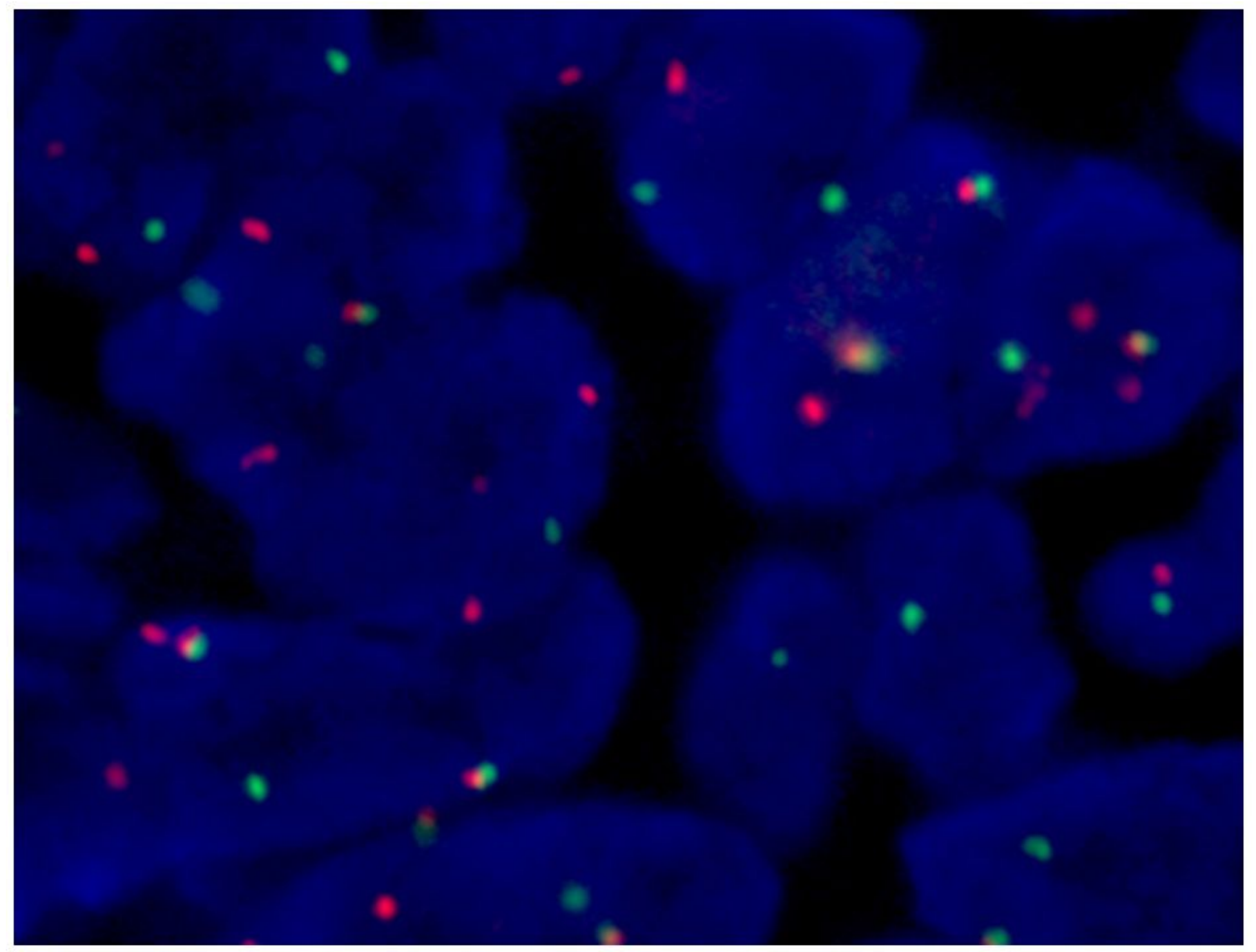

\section{Figure 3}

Representative image of USP6 gene rearrangement analyzed by FISH (X1000). 